\title{
Rediseño de la red de monitoreo atmosférico para control y vigilancia de la calidad del aire en la ciudad de Riobamba
}

\section{Redesign of the atmospheric monitoring network for air quality control and vigilance in the of Riobamba city}

Nelly Patricia Perugachi Cahueñas. ${ }^{1}$; Laura Susana Cocha Telenchana ${ }^{2}$

Recibido: 12-04-2019 / Revisado: 28-05-209 /Aceptado: 28-06-2019/ Publicado: 25-07-2019

\begin{abstract}
DOI: https://doi.org/10.33262/cienciadigital.v3i3.2.723

An Air quality monitoring network is designed for the city of Riobamba, depending on the results obtained through monitoring of pollutants with passive sensors, active sensors, emission characterization and pollutant diffusion models, A redesign is proposed to the monitoring network of atmospheric pollutants for the control and monitoring of air quality, which due to their physical characteristics and commercial and industrial activities has increased their levels of concentration of pollutants, in order to adjust and improve the results that currently has the monitoring network of passive sensors that works in the canton.
\end{abstract}

Key Words. Mathematic models, atmospheric pollutions, atmospheric stability, meteorological parameters.

\section{Resumen}

Se rediseña la red de monitoreo de calidad del aire para la ciudad de Riobamba, en función de los resultados obtenidos mediante monitoreos de contaminantes con sensores pasivos, sensores activos, caracterización de emisiones y modelos de difusión de contaminantes, se propone una red de monitoreo de contaminantes atmosféricos para el control y vigilancia de la calidad del aire, que cubre un mayor territorio, incluyendo nuevas estaciones de monitoreo, con la finalidad de ajustar y mejorar los resultados que actualmente tiene la red de monitoreo de sensores pasivos que funciona en el cantón.

Palabras claves. Calidad del aire, contaminantes atmosféricos, sensores pasivos, red de monitoreo.

1 Escuela Superior Politécnica de Chimborazo, Facultad de Mecánica. Riobamba, Ecuador. patricia.perugachir@espoch.edu.ec

2 Escuela Superior Politécnica de Chimborazo, Sede Orellana, Facultad de Ciencias, Coca, Ecuador, laurasusan_c@yahoo.es 


\section{Introducción}

La contaminación del aire es uno de los principales problemas ambientales de las zonas urbanas en el planeta, tanto en los países desarrollados, por el alto volumen y diversificación de la producción industrial y un flujo intenso de vehículos automotores; como en países en vías de desarrollo por causa del desarrollo no planificado de industrias, uso de tecnologías obsoletas en la producción, servicios y el transporte, la mala calidad del saneamiento básico y el crecimiento urbanístico no planificado [1].

La Ciudad de Riobamba está ubicada en el centro geográfico del país, en la cordillera de los Andes, a 2750 msnm en el centro de la hoya de Chambo, la población según el INEC es de 225.74 habitantes, las actividades económicas más importantes por su situación geográfica, siempre han sido la agricultura y ganadería. Sin embargo, ahora no son las únicas, ya que se debe destacar otras relacionadas al comercio, turismo, educación y ciertas actividades artesanales e industriales [2],. Con este referente y en base a los resultados obtenidos de la investigación se manifiesta que las emisiones de los gases a la atmosfera se deben principalmente a las fuentes móviles y fenómenos naturales como la erosión de los suelos y la erupción del Volcán [3].

El Gobierno Autónomo Descentralizado Municipal del cantón Riobamba mantiene una red primaria básica de monitoreo en el recurso aire desde el año 2008, la misma que inició con la instalación de estaciones de monitoreo pasivo en la zona urbana y áreas de influencia inmediata rural aledaños a la ciudad, esta gestión además fue incidida especialmente por el proceso de erupción del Volcán Tungurahua, con el objetivo de poseer muestras que servirán para plantear medidas para mejorar la calidad de vida de la población del cantón. Como soporte al monitoreo calidad del aire se ha realizado gestiones para mantener convenios de cooperación mutua con la Universidad Nacional de Chimborazo, y con el Distrito Metropolitano de Quito en temas de calidad del aire [ ].

Con el fin de contribuir con la gestión ambiental y sobre la base del monitoreo del aire se ha realizado el proyecto [5], "Emisiones de los gases a la atmosfera y su relación en la calidad del aire de la zona urbana de la ciudad de Riobamba", de los resultados obtenidos y como parte de una herramienta para la gestión y evaluación del aire ambiente en la ciudad se propone un rediseño de la Red de Monitoreo de calidad del aire de la ciudad de Riobamba, el cual permitirá llevar a cabo acciones en forma integral con el fin de controlar, prevenir y/o mejorar de la calidad del aire para preservar la salud de la población [7].

\section{Metodología}

Tomando como referencia los criterios emitidos por el Ministerio de Ambiente que menciona, "En la actualidad, las tres herramientas principales de evaluación de la calidad del aire son: monitoreo del aire ambiente; modelos; inventarios y medición de emisiones" 
[7]. Se propone el rediseño de la Red de Monitoreo de Calidad del Aire de la ciudad de Riobamba, que describa con la mayor exactitud los niveles de contaminación del aire en la ciudad, como medida para actualizar y al mismo tiempo fortalecer la continuidad de las instalaciones de la red de monitoreo de calidad del aire existentes, ampliar su cobertura territorial y representatividad especial, considerando la tendencia de crecimiento poblacional, las observaciones en los tipos de fuentes y según la actividad económica.

Si bien es cierto, Riobamba inicia el monitoreo de calidad del aire desde el año 2008, con tres puntos de monitoreo que hasta el año 2016 únicamente proporciona valores mensuales de $\mathrm{SO}_{2}, \mathrm{NO}_{2}$, como no se cuenta con un registro de la contaminación en forma horaria, diaria que permitan observar picos de mayor contaminación, además no se conocen los datos de otros contaminantes como el $\mathrm{O}_{3}, \mathrm{BTEX}$, pese a que en años anteriores se ha observado que estos inciden en la contaminación del aire sobre todo el Benceno. Es de vital importancia establecer, actualizar una estrategia de monitoreo que proporcione información más a detalle y de las zonas de mayor influencia, como medida de control, de prevención del deterioro del aire ambiente en la ciudad.

De acuerdo a lo mencionado, el rediseño de la red de monitoreo describirá con mayor exactitud los niveles de contaminación del aire en la ciudad, y señala las acciones que de forma integral se ejecutaría entre el GADM Riobamba e Instituciones educativas de nivel Superior, siendo estas factibles para su ejecución y ajustadas a los presupuestos designados, con el objetivo de precautelar la salud de la población, conservando y mejorando la calidad del aire de la ciudad.

El rediseño permitirá dar una continuidad del monitoreo pasivo y ampliar la cobertura territorial su representatividad especial y conocer datos más precisos de monitoreo de calidad del aire con el fortalecimiento de la red de monitoreo atmosférico. Siendo esta una de las herramientas necesarias para la evaluación de la calidad del aire, y, contribuyendo a que se continué con la gestión ambiental que realiza el GAD Municipal de Riobamba realiza para asegurar que las concentraciones de contaminantes se reduzcan o mantengan por debajo de un nivel específico o, en general, aceptables para la protección de la salud humana.

\section{Fundamentación Científico - Técnica}

Una red de monitoreo es el conjunto de estaciones de muestreo, generalmente fijas y continuas, que se establecen para medir los parámetros ambientales necesarios para cumplir con los objetivos fijados y que cubren toda la extensión de un área determinada. Compara regularmente, concentraciones locales de parámetros ambientales con estándares de calidad del aire y las redes establecidas para vigilancia de alertas ambientales permiten implementar acciones en situaciones de emergencia. 
Tomando como base criterios emitidos por la Organización Mundial de la Salud, Organización Panamericana de la Salud principalmente y tomado como referencia los planes de vigilancia de la Calidad del aire en Quito, se contrasta con la realidad de la situación actual que se ha evidenciado en la investigación, de manera de que se plantean que los objetivos sean alcanzables y las actividades ejecutables dentro de un esquema legal e institucional tomando en cuenta el apoyo político requerido.

En este sentido el rediseño está formado en base a las herramientas principales indicadas como necesarias para la evaluación de la calidad del aire y a su vez por la inexistencia de la información requerida para un análisis integral de la gestión del recurso aire. Como consecuente, esto podrá emplearse como el primer referente para la creación de planes operativos en este recurso.

\section{Estrategia para el monitoreo en la ciudad de Riobamba}

Debido a que las decisiones que se tienen que tomar para el diseño de una red de monitoreo dependen fundamentalmente de los objetivos de monitoreo, no existen reglas fijas y fáciles al respecto. A continuación, se presenta recomendaciones para el rediseño de la red de monitoreo de calidad del aire en base a los objetivos establecidos en el siguiente ítem.

\section{Definición de los objetivos del monitoreo atmosférico}

Adquirir valores de contaminación de forma más representativa de la contaminación en la ciudad

- Ubicar estaciones en sectores de influencia

- Determinar la calidad del aire en base a normas y estándares

- Establecer bases para desarrollo de proyectos inherentes al recurso aire

\section{Definición de los parámetros ambientales}

Se consideran los compuestos indicadores de la contaminación atmosférica y los más abundantes establecidos por la OMS, los regulados por la norma nacional y los que se monitorea en otras ciudades a nivel Nacional y estos son: bióxido de azufre (SO2), óxidos de nitrógeno (NOx), ozono $\left(\mathrm{O}_{3}\right)$, monóxido de carbono $(\mathrm{CO})$ y otros contaminantes como benceno, así como partículas sedimentables, $\mathrm{PM}_{10}$ y $\mathrm{PM}_{2,5}$. [10]

Los parámetros ambientales que se tendrán que medir serán los parámetros meteorológicos y topográficos, como: dirección y velocidad del viento, temperatura, diferencial vertical de la temperatura, humedad relativa, precipitación, radiación solar, altitud, tipo de suelo y relieve, factores principales que influyen en la calidad del aire ambiente.

\section{Definición del número y sitios de muestreo}

El diseño de una red de monitoreo está directamente relacionado con la determinación del número y distribución de los sitios de monitoreo y de su frecuencia de muestreo [11]. 
Es necesario mencionar que si bien no existe un método específico para el diseño de la red en la actualidad existen dos enfoques para plantear el mismo. El primer enfoque utiliza un patrón de localización basado en una cuadrícula espacial en donde se muestra información detallada en cuanto a la variabilidad espacial y patrones, resultados de la exposición de contaminantes. Mientras que el segundo, involucra la ubicación de estaciones de monitoreo o sitios de muestreo en lugares representativos, seleccionados cuidadosamente, con base en requerimientos de uso de los datos, este requiere de un número menor de sitios de monitoreo, por lo que es más barato de aplicar. Y es en base a este último enfoque que se procede a rediseñar la red de monitoreo atmosférico en la ciudad de Riobamba [3].

A continuación, se define la localización y determinación del número de estaciones de monitoreo o sitios de muestreo que se considera para cumplir con los objetivos.

\section{Localización de los sitios de muestreo}

Para elegir estas localizaciones se toma en cuenta; tipo de emisiones, fuentes de emisión, los factores topográficos y meteorológicos, información de la calidad del aire.

A continuación, se señala cada uno de ellos:

- Tipo de emisiones y fuentes de emisión: Ya que no se cuenta con un inventario de emisiones, se considera la distribución y tendencia de crecimiento poblacional y las actividades comerciales y zonas observadas de tráfico vehicular.

- Factores topográficos y meteorológicos: Como dirección del viento, temperatura, radiación solar, etc.

- Información de la calidad del aire: El monitoreo atmosférico se lleva a cabo desde el año 2008 en la Ciudad es decir se cuenta con un historial que permite observar tendencias y también en otras ciudades se desarrolla el monitoreo atmosférico lo cual proporcionaría una base de datos contra la cual se pueda comparar y relacionar, además de que indica la calidad del aire del área de estudio. Se considera además otros factores: como uso de suelo, densidad de población.

\section{Resultados}

\section{Descripción general de la zona}

La ciudad de Riobamba es la capital de la provincia de Chimborazo, se encuentra ubicada en el centro geográfico del Ecuador, en la cordillera de los Andes, a $1^{\circ} 40^{\prime} 28^{\prime \prime} \mathrm{S} 78^{\circ} 38^{\prime} 54^{\prime \prime} \mathrm{O}$ y a una altura de $2750 \mathrm{~m} \mathrm{~s}$. n. m., en el centro de la hoya de Chambo, con una población de 350000 habitantes, en su zona metropolitana, 
una de las más pobladas del país. La superficie delimitada por el perímetro urbano de la ciudad es de aproximadamente $45 \mathrm{~km}^{2}$, está en una zona de topografía plana - meseta, se los conoce con el nombre de basalto conformada por productos piroclásticos, con una temperatura promedio anual de $12{ }^{\circ} \mathrm{C}$, con pocas variaciones a lo largo del año por su posición geográfica $[12,13]$.

Figura. 1 Mapa físico de ciudad de Riobamba.

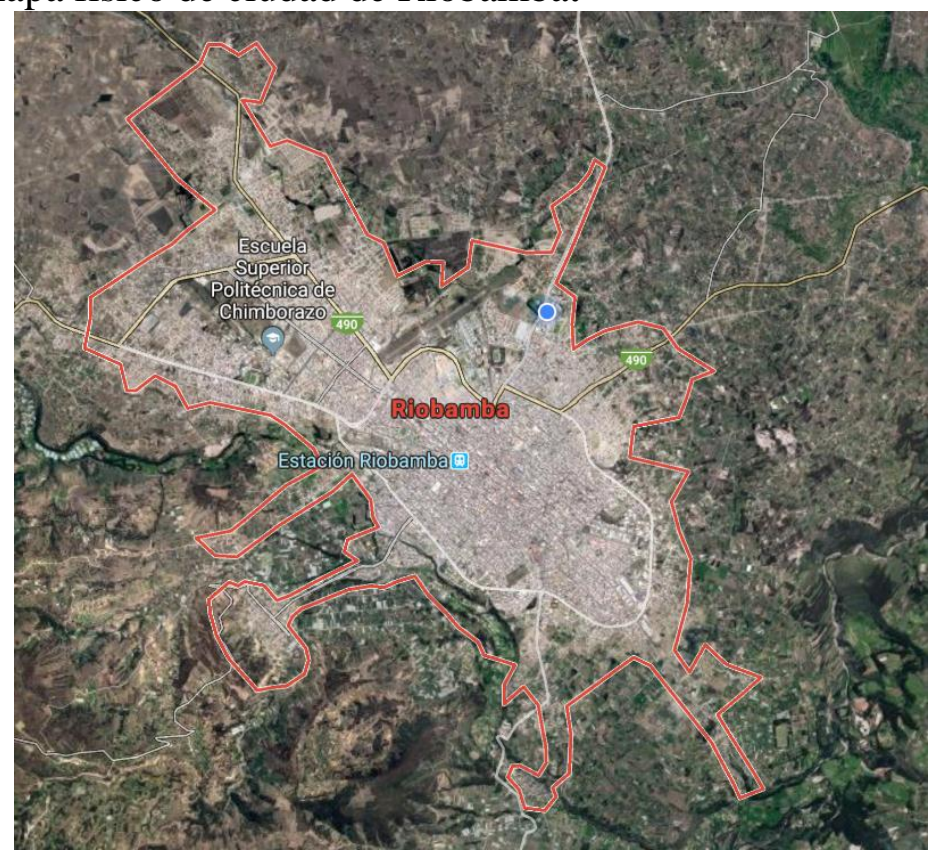

Figura 2. Uso del Suelo de la ciudad de Riobamba.

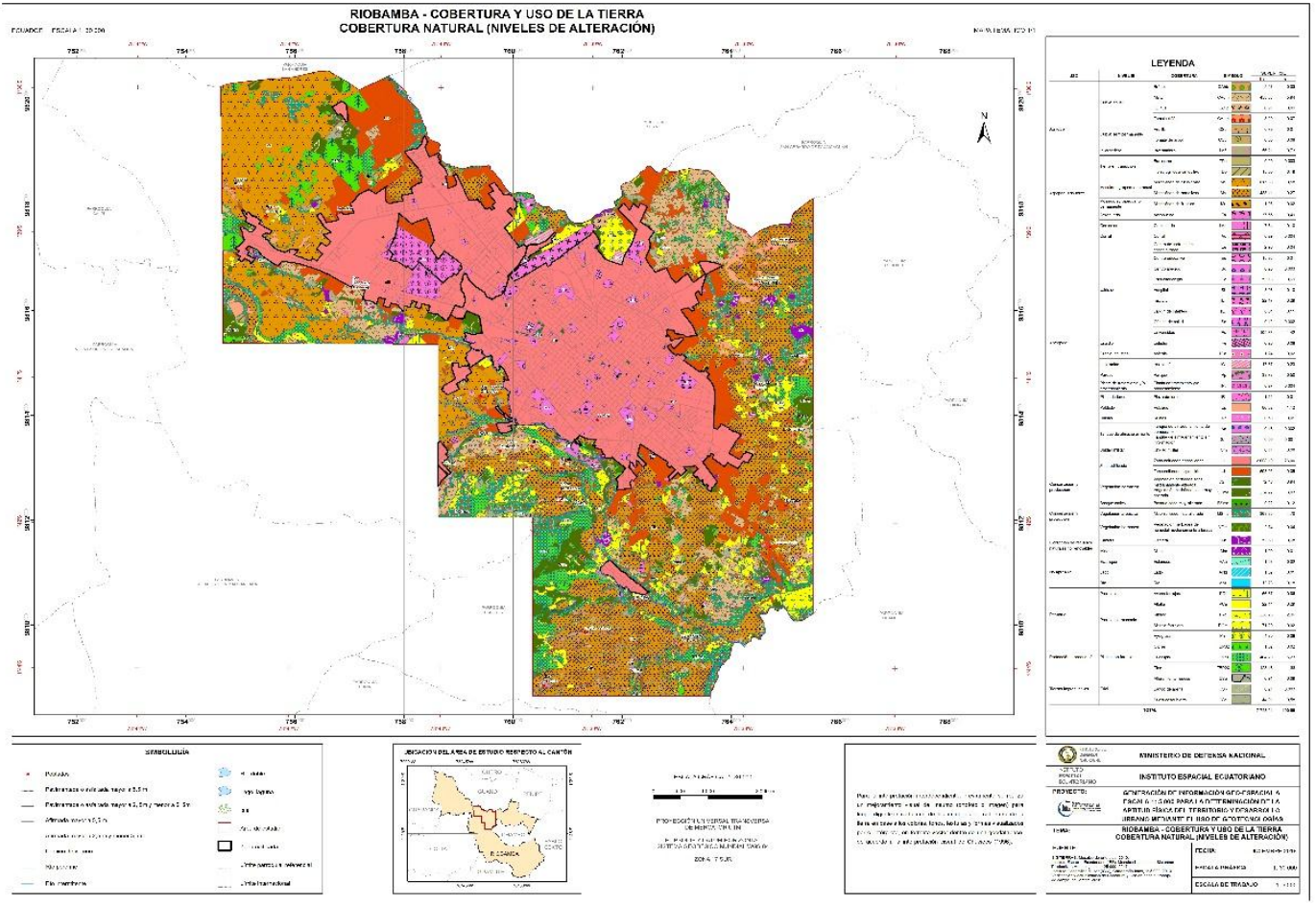




\section{Datos Meteorológicos}

Figura. 3 Datos de dirección del viento (Datos, Estación Meteorológica del Grupo de Energías Alternativas de la ESPOCH).

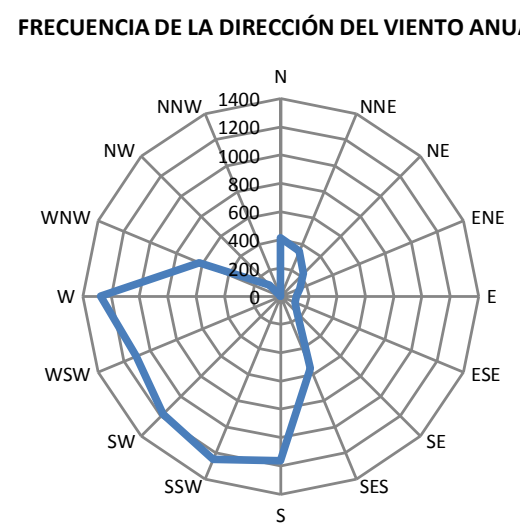

La dirección predominante del viento esta hacia entre el sur y oeste, de acuerdo a los resultados de la Figura 3.

Figura. 4 Temperatura promedio anual por días (Datos, Estación Meteorológica del Grupo de Energías Alternativas de la ESPOCH).

TEMPERATURA PROMEDIO ANUAL POR HORAS

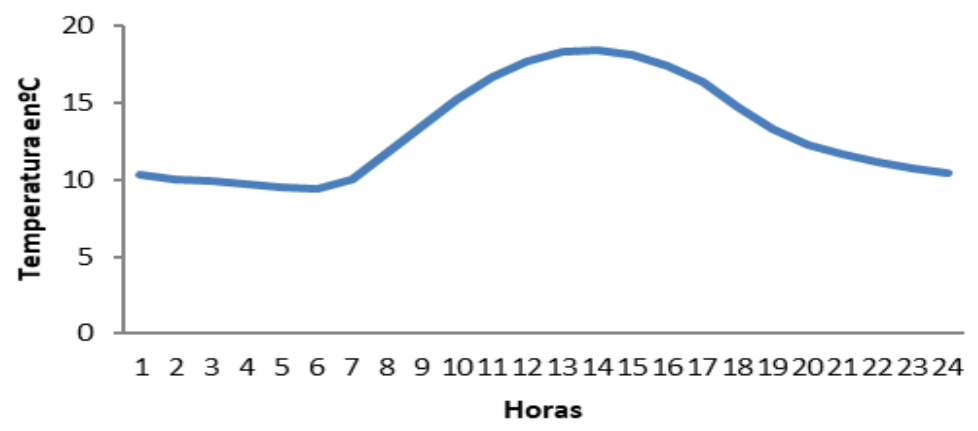

Figura. 5 Velocidad del viento anual por horas (Datos, Estación Meteorológica del Grupo de Energías Alternativas de la ESPOCH).

VELOCIDAD DEL VIENTO PROMEDIO ANUAL POR

HORAS

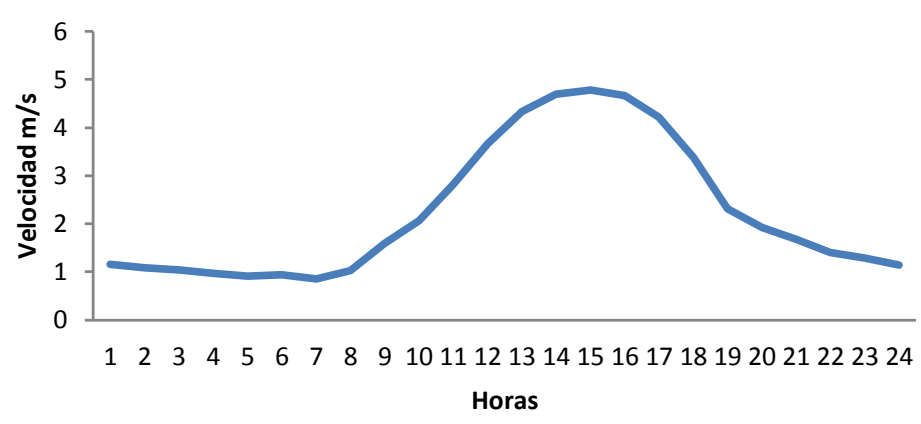


En las Figuras 4 y 5, muestran el comportamiento de la temperatura y la velocidad del viento promedio horas, en las que se puede observar una tendencia regular con temperaturas y velocidades del viento más altas a las horas de la tarde, con las variaciones más significativas entre las 9:00 y 18:00, debido a su posición geográfica las mismas se mantienen a lo largo del año (Amanda H., et al, 2006),

\section{Monitoreo de la calidad del aire}

Ubicación de la red de monitoreo

Figura. 6 Zona de estudio y localización de los puntos de monitoreo pasivo de calidad del aire del GAD Riobamba. (Los datos como coordenadas geográficas, orografía, red vial son recogidos del Instituto Geográfico Militar).

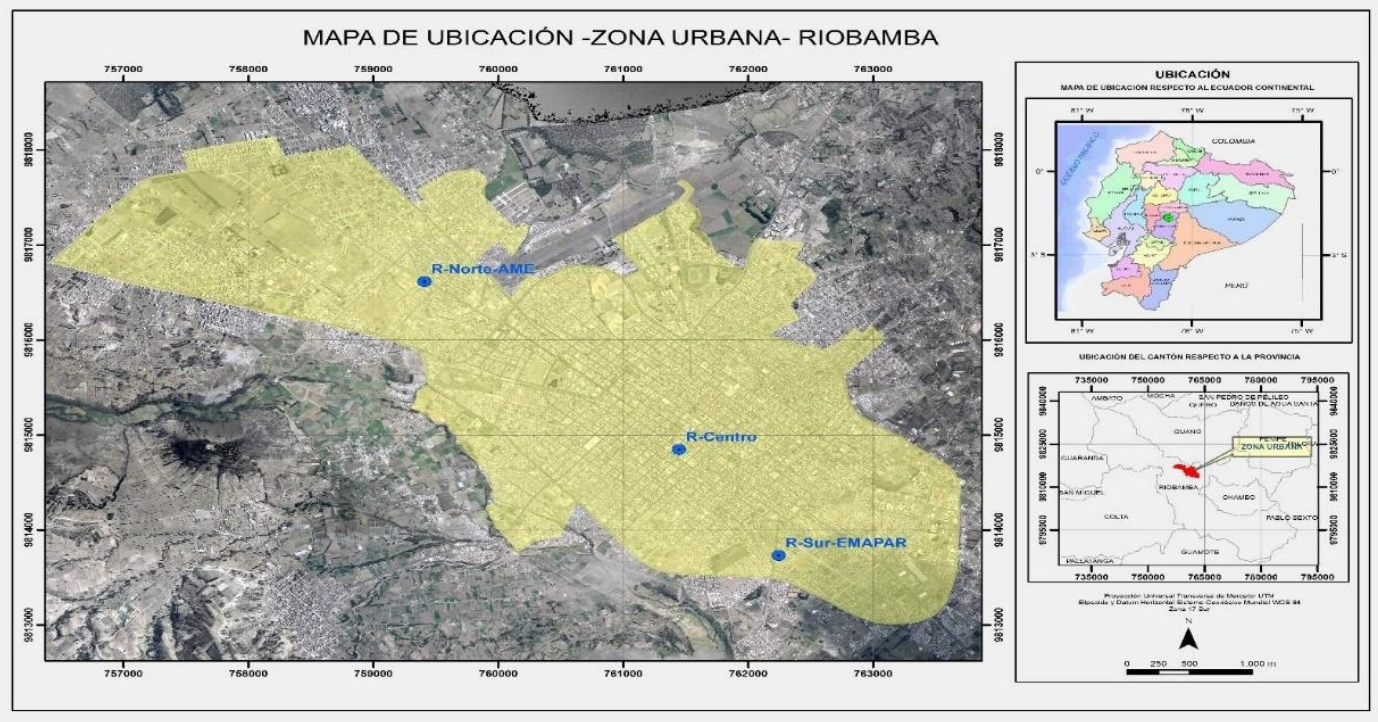

Nota: Ubicación geográfica de la zona de estudio y localización de las estaciones de monitoreo de calidad del aire, al noroeste (R-Norte AME), al centro (R-Centro) y al sureste (R-Sur-EMAPAR) (Susana Cocha 2017)

Como se observa en la Figura 6, actualmente existen tres estaciones de monitoreo ubicados en el área urbana de norte a sur de la ciudad, codificados como: R-NorteAME; R-Centro y R-Sur-EMAPAR.

Mensualmente se obtiene una concentración de los gases dióxido de azufre, dióxido de nitrógeno por medio del método de monitoreo pasivo. Análisis de datos de dióxido de azufre (SO2).

\section{Análisis de concentración de dióxido de azufre (SO2)}

Para el análisis se tiene presente que el dióxido de azufre se descarga a la atmosfera principalmente de las emisiones de industria, fuentes móviles y por las emisiones de los volcanes activos. 
Figura.7 Promedio de concentración de dióxido de azufre (SO2) en las estaciones de monitoreo.

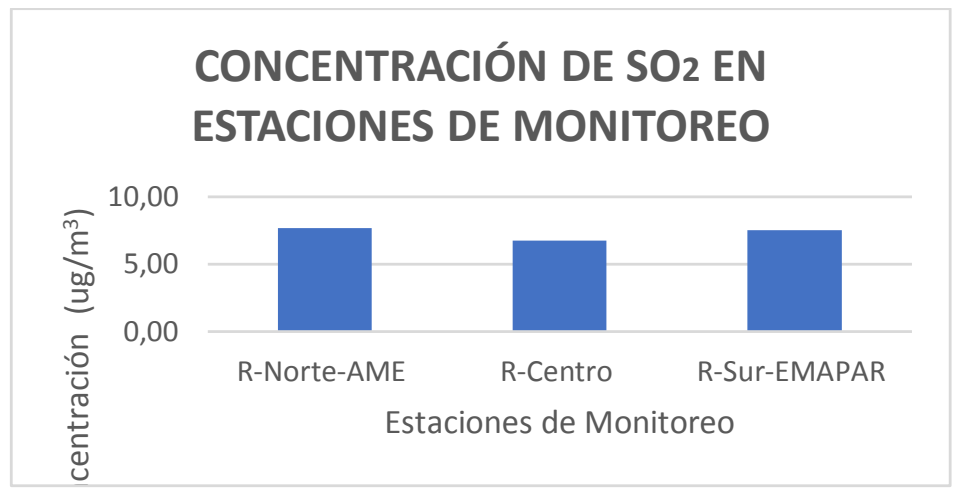

En la Figura 7 se muestra la concentración promedio del SO2 en las diferentes estaciones de monitoreo, presentando una pequeña variación entre las ubicadas en el centro norte y sur, siendo mayor en estas dos últimas,

\section{Comparación de las concentraciones de dióxido de azufre (SO2), con la Norma y Guías de la OMS}

Para conocer si existe violación de Norma se compara el resultado promedio anual de cada estación como se muestra en la Tabla 1.

Tabla. 1 Comparación de SO2 ( $\mu \mathrm{g} / \mathrm{m} 3)$ con la Norma y Guías de la OMS.

\begin{tabular}{lllllll}
\hline AÑO & $\begin{array}{l}\text { R-Norte- } \\
\text { AME }\end{array}$ & $\begin{array}{l}\text { R- } \\
\text { Centro }\end{array}$ & $\begin{array}{l}\text { R-Sur- } \\
\text { EMAPAR }\end{array}$ & TULSMA & OMS & $\begin{array}{l}\text { EXECEDE } \\
\text { LOS } \\
\text { LIMITES }\end{array}$ \\
\hline $\mathbf{2 0 0 8}$ & 7,93 & 5,95 & 6,03 & 60 & 50 & NO \\
$\mathbf{2 0 0 9}$ & 13,96 & 7,57 & 9,88 & 60 & 50 & NO \\
$\mathbf{2 0 1 0}$ & 8,92 & 8,71 & 8,88 & 60 & 50 & NO \\
$\mathbf{2 0 1 4}$ & 4,69 & 5,34 & 4,16 & 60 & 50 & NO \\
$\mathbf{2 0 1 5}$ & 6,35 & 4,79 & 6,14 & 60 & 50 & NO \\
$\mathbf{2 0 1 6}$ & 4,39 & 8,16 & 10,03 & 60 & 50 & NO \\
\hline
\end{tabular}

Nota: Comparación de las concentraciones de dióxido de azufre anual y por estación con Normas y Guías de Calidad del Aire (Cocha, 2017).

Como se observa en la Tabla 1., los niveles más altos de contaminación obtenidos, se señala de la siguiente manera: $13,96 \mu \mathrm{g} / \mathrm{m}^{3}$ que se registró en el año 2009 en la estación RNorte-Ame, y en el año 2016 con un promedio de $10,03 \mu \mathrm{g} / \mathrm{m}^{3}$, en la Estación R-SurEMAPAR, estos son los valores más alto de todo el tiempo de monitoreo.

El promedio de $\mathrm{SO}_{2}$ en todos los años de monitoreo en las estaciones Norte, Centro y Sur de la zona urbana de la ciudad de Riobamba no registra un índice mayor al establecido en la Norma Ecuatoriana de Calidad del Aire, ni supera los estándares establecidos en las Guías de la Organización Mundial para la Salud. 
Según los datos del segundo periodo de monitoreo los niveles de concentración de este gas disminuyen en comparación a los valores registrados en el primer período, posiblemente por influencia de los episodios de erupción del Volcán Tungurahua que se manifestaron (Boletines especiales del volcán Tungurahua No. 01 de enero y el No.

05 de septiembre del 2009, publicados por el Instituto Geofísico).

Otra razón puede ser, por el uso de combustible que se comercializa, de mejor calidad es decir de mayor octanaje a partir del año 2012, en este año se anunció una mejora en la calidad de la gasolina extra de 81 a 87 octanos, mientras que la súper pasaba de 90 a 92 octanos (Ministerio de Hidrocarburos).

\section{Análisis de concentración de nitrógeno (NO2)}

Figura. 8 Promedio de concentración del NO2 en las estaciones de monitoreo.

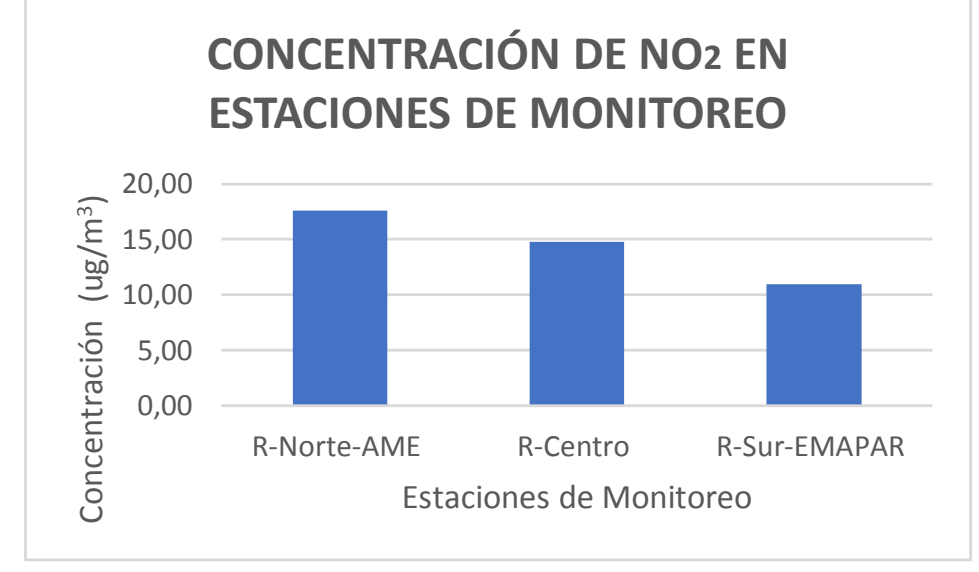

Resultados de la concentración de $\mathrm{NO}_{2}$ registrado en las estaciones de monitoreo, donde se observa mayor concentración en la estación Norte-AME, que puede ser por la incidencia del trafico vehicular.

Comparación de las concentraciones de dióxido de nitrógeno (NO2), con la Norma y Guías de la OMS.

Para conocer si existe violación de Norma se compara el resultado promedio anual de cada estación como se muestra en la Tabla 2.

Tabla. 2 Comparación de NO2 $(\mu \mathrm{g} / \mathrm{m} 3)$ con Norma Nacional y Guía Internacional.

\begin{tabular}{llllll}
\hline AÑO & $\begin{array}{l}\text { R- } \\
\text { Norte- } \\
\text { AME }\end{array}$ & $\begin{array}{l}\text { R- } \\
\text { Centro }\end{array}$ & $\begin{array}{l}\text { R-Sur- } \\
\text { EMAPAR }\end{array}$ & $\begin{array}{l}\text { NORMA } \\
\text { NACIONAL y } \\
\text { OMS }\end{array}$ & $\begin{array}{l}\text { EXECEDE LOS } \\
\text { LíMITES }\end{array}$ \\
\hline $\mathbf{2 0 0 8}$ & 12,94 & 14,28 & 9,30 & 40 & NO \\
$\mathbf{2 0 0 9}$ & 17,90 & 16,71 & 14,98 & 40 & NO \\
$\mathbf{2 0 1 0}$ & 17,69 & 19,72 & 14,34 & 40 & NO \\
$\mathbf{2 0 1 4}$ & 17,74 & 17,08 & 13,15 & 40 & NO \\
$\mathbf{2 0 1 5}$ & 28,77 & 12,79 & 7,15 & 40 & NO \\
$\mathbf{2 0 1 6}$ & 10,57 & 8,23 & 6,77 & 40 & NO \\
\hline
\end{tabular}


Nota: Comparación de las concentraciones de dióxido de nitrógeno anual y por estación con Normas y Guía de Calidad del aire

La Tabla 2 muestra que en el período de monitoreo (2008-2010), presentan promedios más elevados que el segundo período de monitoreo (2014-2016). En el año 2015 se registra la mayor concentración de dióxido de nitrógeno $\left(\mathrm{NO}_{2}\right)$ en la estación R-Norte-AME que es de $28,77 \mu \mathrm{g} / \mathrm{m}^{3}$. Esta estación registra los más altos niveles de $\mathrm{NO}_{2}$.

El promedio de $\mathrm{NO}_{2}$ en todos los años de monitoreo en las estaciones Norte, Centro y Sur de la de la zona de estudio, no supera el límite permisible de $40 \mu \mathrm{g} / \mathrm{m}^{3}$ estipulado en la Norma de Calidad del Aire y en las Guías de la Organización Mundial de la Salud.

\section{Análisis de las concentraciones del $\operatorname{Ozono}\left(\mathrm{O}_{3}\right)$}

El ozono troposférico $\left(\mathrm{O}_{3}\right)$ se forma por reacciones químicas en el aire entre los hidrocarburos y los óxidos de nitrógeno bajo la influencia de la luz solar.

Figura. 9 Concentración de Ozono (O3) $\mu \mathrm{g} / \mathrm{m}^{3}$.

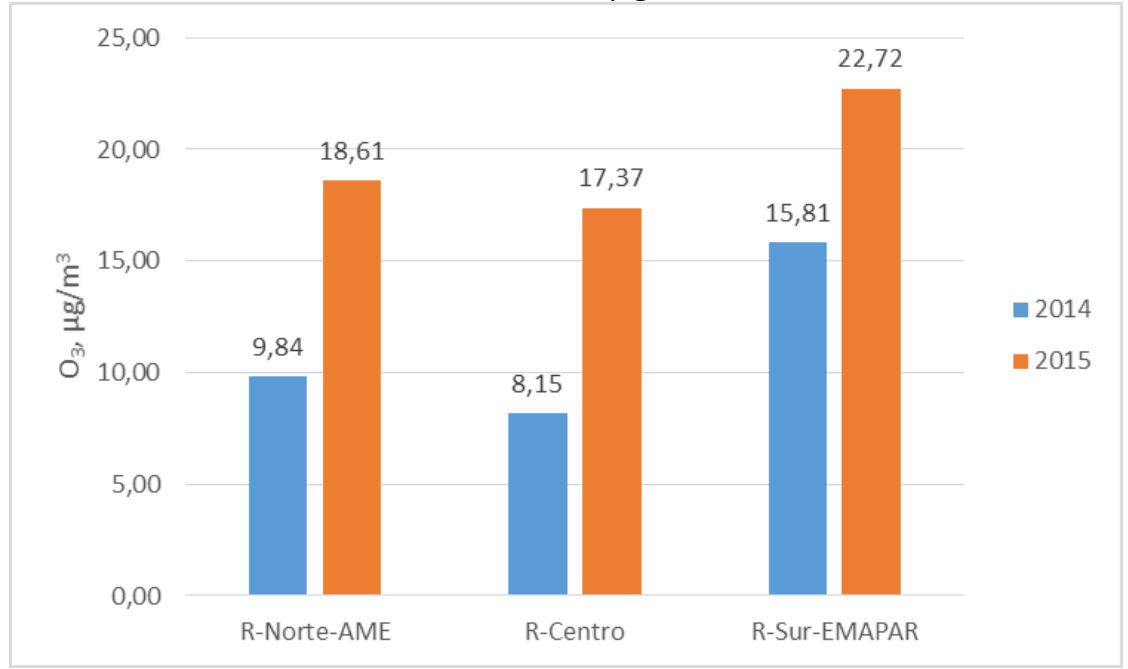

Como se observa en la Figura 9, este parámetro registra en el año 2014 un promedio de $11,27 \mu \mathrm{g} / \mathrm{m}^{3}$, mientras que en el año 2015 presento una concentración de $19,57 \mu \mathrm{g} / \mathrm{m}^{3}$. La concentración más alta se presentó en la estación R-Sur-EMAPAR con un valor de 22,72 $\mu \mathrm{g} / \mathrm{m}^{3}$ en el año 2015.

Para este contaminante existe un límite máximo establecido por la Norma de calidad del Aire octohorario que es $100 \mu \mathrm{g} / \mathrm{m}^{3}$, razón por la cual no se compara con el nivel promedio registrado para este contaminante ya que el valor es mensual. Sin embargo los valores encontrados están muy por debajo del límite máximo permisible octohorario.

\section{Análisis de las concentraciones del benceno, tolueno, xileno}

Las emisiones se deben principalmente al tráfico vehicular, por autos a gasolina, se produce por la combustión incompleta de los combustibles, el aceite lubricante del motor y adicionalmente por la evaporación de los combustibles del sistema del vehículo, esto ocurre 
cuando se realiza una conducción alborotada o luego de finalizar la conducción cuando el vehículo está caliente. Otra fuente de emisión es la erupción de volcanes.

Figura. 10 Concentraciones del benceno, tolueno, xileno $\mu \mathrm{g} / \mathrm{m} 3$ en las estaciones de monitoreo.

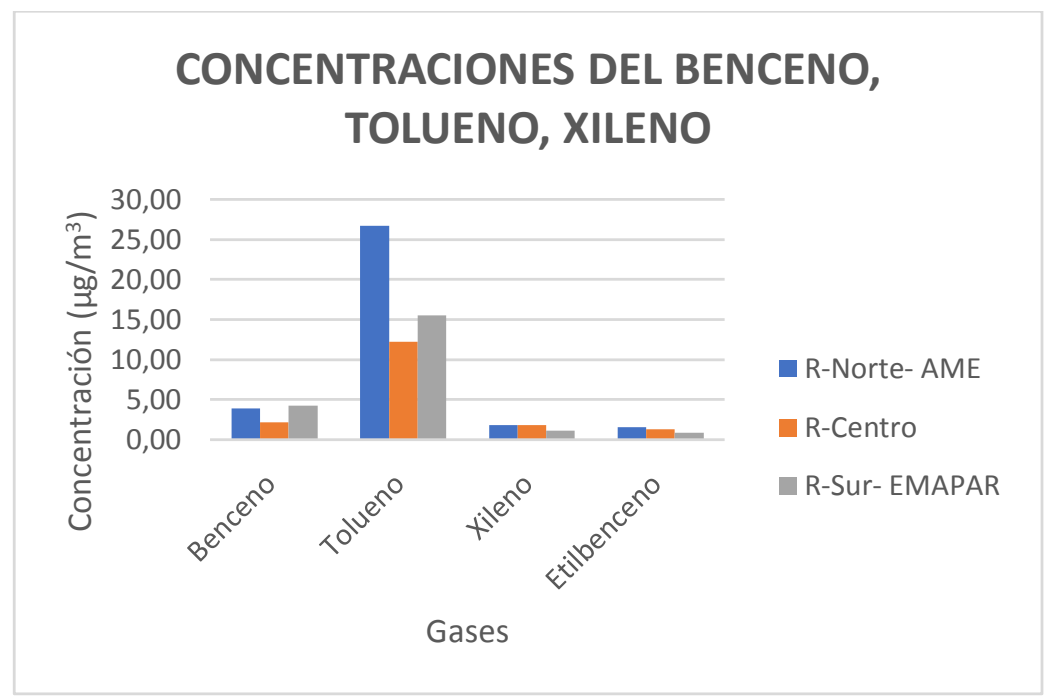

Se muestra que en el año 2009 se superó el límite permisible de benceno en la estación RNorte-AME con un valor de $6,86 \mu \mathrm{g} / \mathrm{m}^{3}$ y $8,36 \mu \mathrm{g} / \mathrm{m}^{3}$ en la estación R-SurEMAPAR. Pudo deberse a las emisiones producidas por el parque automotor ya que en esta zona se registró una mayor concentración tanto de dióxido de azufre como de dióxido de nitrógeno en el mismo año y a las emisiones producidas por la actividad del volcán Tungurahua.

Se muestra que el touleno, de acuerdo a los valores guía para contaminantes del aire no cancerígenos recomendados por la OMS que es de $260 \mu \mathrm{g} / \mathrm{m}^{3}$ sobre los cuales hay efectos sobre el Sistema Nervioso Central de los trabajadores en un tiempo de exposición de una semana, no registra superación de valores en ningún año.

Se muestra que el xileno, de acuerdo a los valores guía para contaminantes del aire no cancerígenos recomendados por la OMS que es $870 \mu \mathrm{g} / \mathrm{m}^{3}$ sobre los cuales hay efectos en la salud en un tiempo de exposición de un año, no se observa superación.

Según los valores guía para contaminantes no cancerígenos recomendados por la OMS, el etilbenceno tiene como efectos sobre la salud el incremento de peso de los órganos, el valor de concentración tolerable $22.000 \mu \mathrm{g} / \mathrm{m}^{3}$ en un tiempo promedio de exposición de 1 año y de acuerdo a los valores registrados en todo el período monitoreado no existe excedencia de este contaminante [15].

\section{Difusión de contaminantes}

Para el estudio de difusión de contaminantes se aplica el modelo ISCLT usando datos meteorológicos recolectados en la estación meteorológica de la ESPOCH y datos de emisiones de fuente relativos, para calcular la distribución relativa del contaminante bajo las condiciones de la zona [16-18]. 
Figura. 11 Distribución del contaminante alrededor de la fuente.

\section{DISTRIBUCIÓN DE CONCENTRACIÓN ALREDEDOR DE LA FUENTE}

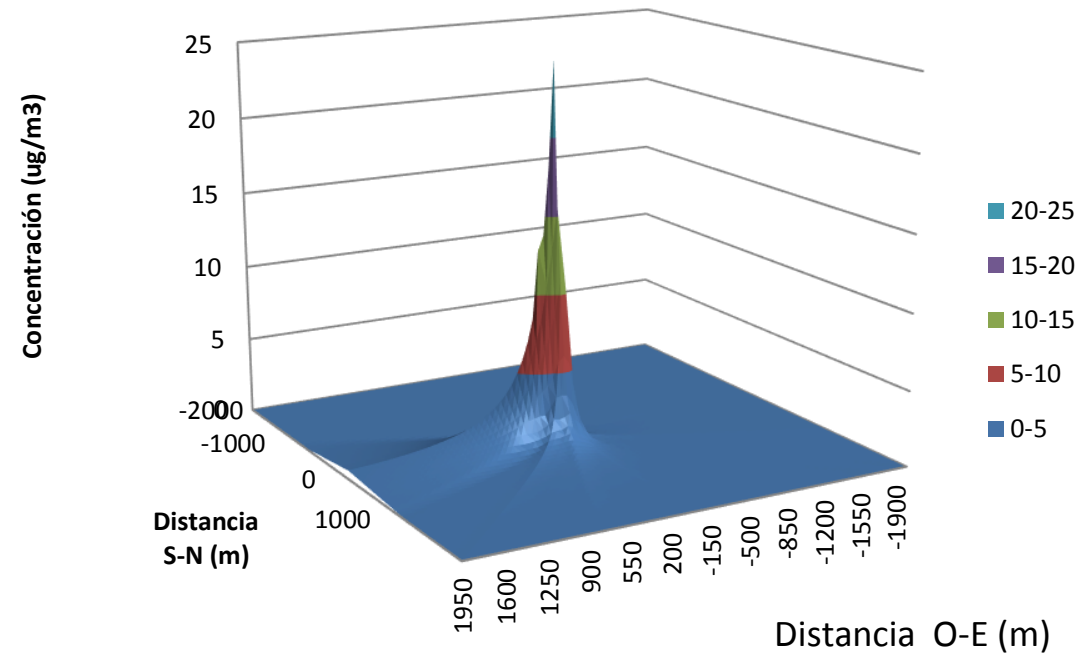

Figura. 12 Puntos donde se ubican los 10 máximos de concentración alrededor de la fuente.

\section{MÁXIMOS DE CONCENTRACIÓN}

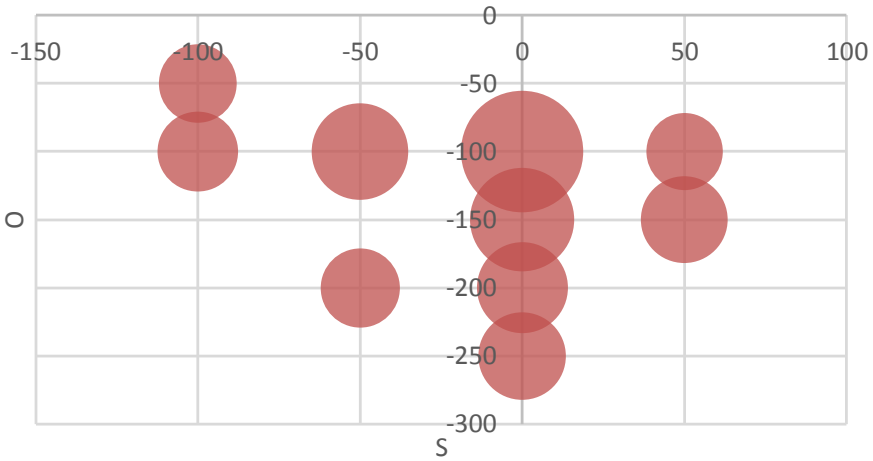

En la Figura 11 se observa la distribución relativa de los contaminantes calculadas con el modelo ISCLT (Aeromod, EPA, 2004), bajo las condiciones físicas y meteorológicas de la zona y en la Figura 12, se observa los máximos de concentración, los cuales se dirigen principalmente hacia el sur oeste, dado la predominancia de la dirección del viento en esa dirección, razón por la cual hay más puntos de máximo en la misma, cabe recalcar que el sistema tomado en nuestro caso para representar estos puntos es hacia el sur oeste por la dirección del viento [12].

\section{Discusión}

En base a los resultados se propone la ubicarán adicionalmente a la red actual 4 puntos en zonas de influencia por actividades industriales, asentamiento poblacional y tráfico vehicular. El rediseño de la red finalmente estaría conformado por siete puntos o estaciones. La posible ubicación de estas se muestra en la Figura 13. 
Figura 13. Propuesta de rediseño de la red de monitoreo atmosférico en Riobamba.

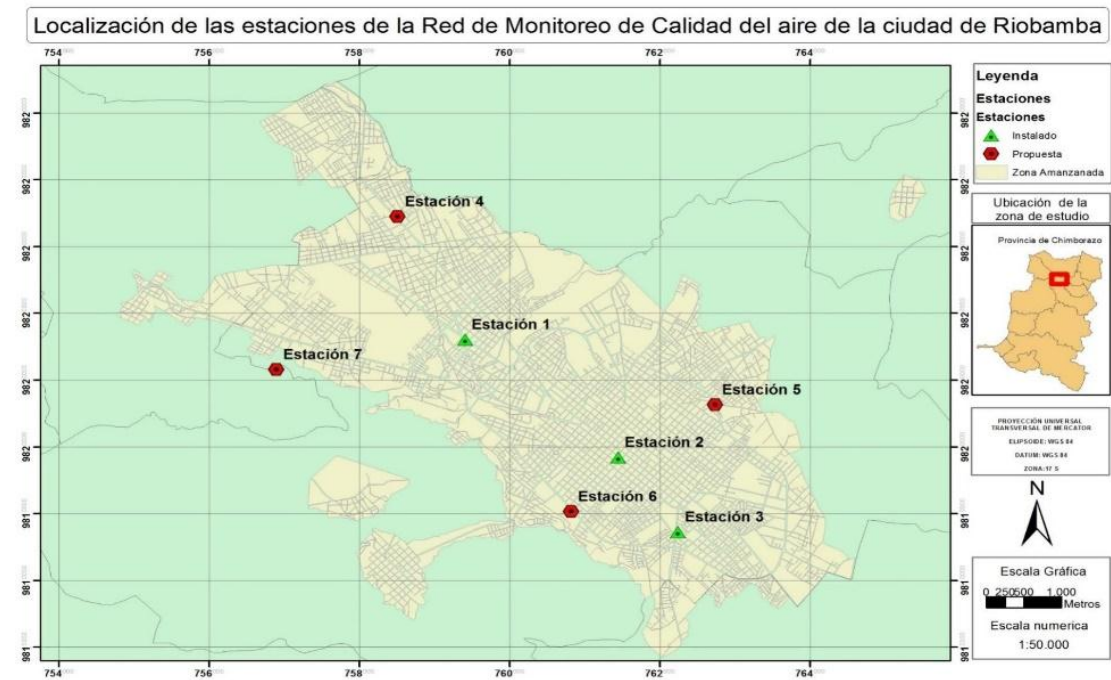

\section{Densidad o número de puntos de muestreo requeridos}

El número final de estaciones seleccionado se elige en función de la problemática existente en el área o sector, el tipo de zona, los recursos económicos, humanos y tecnológicos disponibles. También es considerada la necesidad de contar con estaciones libres de influencias urbanas, que se caractericen como limpias o de fondo (Estación 7) en términos de la difusión de los contaminantes determinados por el modelo.

Considerando los criterios antes mencionados se recomienda ampliar la red de monitoreo ubicando cuatro puntos de monitoreo que se sumaran a los tres existentes para un total de siete sitios colocados en zonas residenciales comerciales de alto tráfico vehicular, de expansión poblacional y de industria Tabla 3.

De igual manera se recomienda la implementación de al menos una estación automática para tener datos continuos en el tiempo y poder correlacionarles con los de las estaciones de sensores pasivos, cumpliendo las normas de la OPS.

Tabla 3. Número de estaciones propuestas de muestreo de la calidad del aire

\begin{tabular}{ll}
\hline Estación & Descripción del área \\
\hline $\mathbf{1}$ & Zona de alta circulación vehicular, zona educativa y residencial \\
$\mathbf{2}$ & Zona Comercial, de tráfico de vehicular (Estación automática) \\
$\mathbf{3}$ & Zona residencial y comercial \\
$\mathbf{4}$ & Zona residencial, suelos sin cobertura vegetal \\
$\mathbf{5}$ & Desarrollo urbano, actividades industriales, tráfico vehicular \\
$\mathbf{6}$ & Residencial y comercial \\
$\mathbf{7}$ & Residencial, zona de prueba para comparar con la parte urbana \\
\hline
\end{tabular}

Nota: Descripción de las estaciones sugeridas para ampliar la red de monitoreo actual Elaborado por: Investigador, 2017 


\section{Requerimientos del sitio de muestreo}

Algunas de las recomendaciones que se fijan para este tema se extraen de los documentos de la OPS, que recoge información de manuales de organismos como la EPA y del Código Federal de Regulaciones de los EE.UU. (U.S. Code of Federal Regulations) (40 CFR 58)Los requerimientos generales de ubicación.

A continuación se presenta los criterios a considerar para selección del sitio.

$\checkmark$ El sitio debe tener fácil acceso debido a que se tendrán que realizar visitas regulares al mismo para recolectar muestras, inspeccionarlo; calibrarlo o para su mantenimiento (estaciones automáticas y activas).

$\checkmark$ A su vez deberá estar protegido de posibles actos de vandalismo u otros que alteren la toma de muestras. El sitio debe contar con la infraestructura necesaria como electricidad y líneas de teléfono para poder operar cualquier tipo de equipo de muestreo en el sitio. En caso de muestreadores automáticos y activos.

$\checkmark$ No podrá haber obstáculos que afecten el movimiento del aire en el sitio, ni fuentes de emisión que puedan invalidar las muestras por el arrastre a la toma del muestreador de las emisiones de alguna fuente. Es decir, el movimiento del aire alrededor de la entrada de la toma de muestra deberá estar libre de restricciones que afecten el flujo del aire en las cercanías del muestreador, por lo que se recomienda ubicarlo algunos metros alejado de edificios, balcones, árboles, etc.

A continuación se da a conocer las distancias recomendadas de ubicación:

$\checkmark$ Para asegurar un flujo lo más libre posible, se deben evitar árboles y edificios en un área de $10 \mathrm{~m}$., alrededor del sitio de muestreo y no tomar muestras en las superficies laterales de los edificios.

$\checkmark$ Para minimizar los efectos de las fuentes locales, se recomienda instalar la estación de monitoreo a una distancia de por lo menos 20 metros de cualquier fuente industrial, doméstica o de carreteras con alto tráfico.

$\checkmark$ La entrada al muestreador debe estar entre 1,5 y $4 \mathrm{~m}$., sobre el nivel del piso. Existen algunas circunstancias, para los estudios de los antecedentes de contaminación en ciudades, en donde no es posible cumplir con el requisito de una altura de $4 \mathrm{~m}$., por lo cual se han realizado toma de muestra hasta $8 \mathrm{~m}$.

$\checkmark$ La entrada al muestreador no debe localizarse cerca de fuentes de contaminación, para evitar arrastres de plumas de chimeneas domésticas o industriales 
$\checkmark$ Para medir los parámetros meteorológicos se recomienda instalar los instrumentos a una altura mínima de $10 \mathrm{~m}$. sobre el nivel del suelo, y tomar mediciones a diferentes alturas con el objeto de obtener gradientes térmicos.

\section{Determinación de tiempos de muestreo}

Duración del Programa: se recomienda como la duración del muestreo a los 12 meses que abarca un año completo del período de evaluación, porque de esta manera se toma en cuenta la injerencia de los cambios estacionales y porque la Norma de Calidad del aire emite un límite máximo de concentración de un contaminante específico por año.

Tabla 4. Período de medición.

\begin{tabular}{|c|c|c|}
\hline Contaminante & Valor* & Periodo de medición \\
\hline $\begin{array}{l}\text { Partículas } \\
\text { sedimentables }\end{array}$ & $\begin{array}{l}1 \mathrm{mg} / \mathrm{cm}^{2} \mathrm{~d} \\
30 \text { días }\end{array}$ & $\begin{array}{l}\text { eColectada durante } 30 \text { (treinta) días de } \\
\text { forma continua }\end{array}$ \\
\hline $\begin{array}{l}\text { Material particul } \\
\text { menor a } 10 \text { micro }\end{array}$ & nes & $\begin{array}{l}\text { Promedio aritmético de todas las muestras } \\
\text { colectadas en } 1 \text { año }\end{array}$ \\
\hline$\left(\mathrm{PM}_{10}\right)$ & $100 \mu \mathrm{g} / \mathrm{m}^{3}$ & $\begin{array}{l}\text { Promedio aritmético de todas las muestras } \\
\text { colectadas en } 24 \text { horas. }\end{array}$ \\
\hline $\begin{array}{l}\text { Material particul } \\
\text { menor a } 2,5 \text { micro }\end{array}$ & do $15 \mu \mathrm{g} / \mathrm{m}^{3}$ & $\begin{array}{l}\text { Promedio aritmético de todas las muestras } \\
\text { colectadas en un año }\end{array}$ \\
\hline$\left(\mathrm{PM}_{2,5}\right)$ & $50 \mu \mathrm{g} / \mathrm{m}^{3}$ & $\begin{array}{l}\text { Promedio aritmético de todas las muestras } \\
\text { colectadas en } 24 \text { horas. }\end{array}$ \\
\hline $\begin{array}{l}\text { Dióxido de az } \\
\text { (SO2) }\end{array}$ & $\mathrm{re} 125 \mu \mathrm{g} / \mathrm{m}^{3}$ & $\begin{array}{l}\text { Concentración } 24 \text { horas en de todas las } \\
\text { muestras colectadas. }\end{array}$ \\
\hline & $500 \mu \mathrm{g} / \mathrm{m}^{3}$ & $\begin{array}{l}\text { Concentración en un periodo de } 10 \\
\text { minutos de todas las muestras colectadas }\end{array}$ \\
\hline & $60 \mu \mathrm{g} / \mathrm{m}^{3}$ & $\begin{array}{l}\text { Promedio aritmético de todas las muestras } \\
\text { colectadas en } 1 \text { año }\end{array}$ \\
\hline Monóxido & $\operatorname{de} 10 \mu \mathrm{g} / \mathrm{m}^{3}$ & Concentración en 8 horas consecutivas \\
\hline carbono $(\mathrm{CO})$ & $30 \mu \mathrm{g} / \mathrm{m}^{3}$ & Concentración máxima en 1 hora \\
\hline Ozono $\left(\mathrm{O}_{3}\right)$ & $100 \mu \mathrm{g} / \mathrm{m}^{3}$ & Concentración en 8 horas consecutivas \\
\hline $\begin{array}{l}\text { Dióxido de nitróg } \\
\text { (NO2) }\end{array}$ & $1040 \mu \mathrm{g} / \mathrm{m}^{3}$ & $\begin{array}{l}\text { Promedio aritmético de todas las muestras } \\
\text { colectadas en } 1 \text { año }\end{array}$ \\
\hline & $200 \mu \mathrm{g} / \mathrm{m}^{3}$ & $\begin{array}{l}\text { Concentración máxima en } 1 \text { hora de todas } \\
\text { las muestras colectadas }\end{array}$ \\
\hline Benceno & $5 \mu \mathrm{g} / \mathrm{m}^{3} \mu \mathrm{g} / \mathrm{m}^{3}$ & $\begin{array}{l}\text { Promedio aritmético de todas las muestras } \\
\text { colectadas en } 1 \text { año }\end{array}$ \\
\hline
\end{tabular}

*Los valores están sujetos a las condiciones de referencia de $25^{\circ} \mathrm{C}$ y $760 \mathrm{~mm} \mathrm{Hg}$.

Nota: Se muestra el periodo de medición para el cual la autoridad ambiental de control establece un límite máximo permitido por contaminante. Fuete: Norma de Calidad del Aire 
Frecuencia: La frecuencia de monitoreo está determinado por el tipo de método o técnica de muestreo que debe estar en relación a los criterios mencionados en la Normativa Ecuatoriana de Calidad del Aire, la misma que establece un período de medición en promedios diario, mensual, anual y concentraciones horarias y señala el límite máximo permisible por contaminante. Se muestra en la tabla 4.

\section{Selección del equipo o técnica de monitoreo y método de medición}

Se toma en cuenta, el costo del instrumento y su complejidad contra su confiabilidad y su funcionamiento. Y principalmente por el cumplimiento de los objetivos planteados y los antecedentes de los métodos de análisis que en ciudades País se utiliza, se recomienda por tanto los métodos de muestreo: pasivos, activo y automático, este último permitirá vigilar picos de concentraciones y alertar a la población. Estas técnicas se describen en la tabla 5, y resume ventajas y desventajas como la inversión estimada del costo de adquisición de cada uno.

Tabla 5. Técnicas de monitoreo atmosférico.

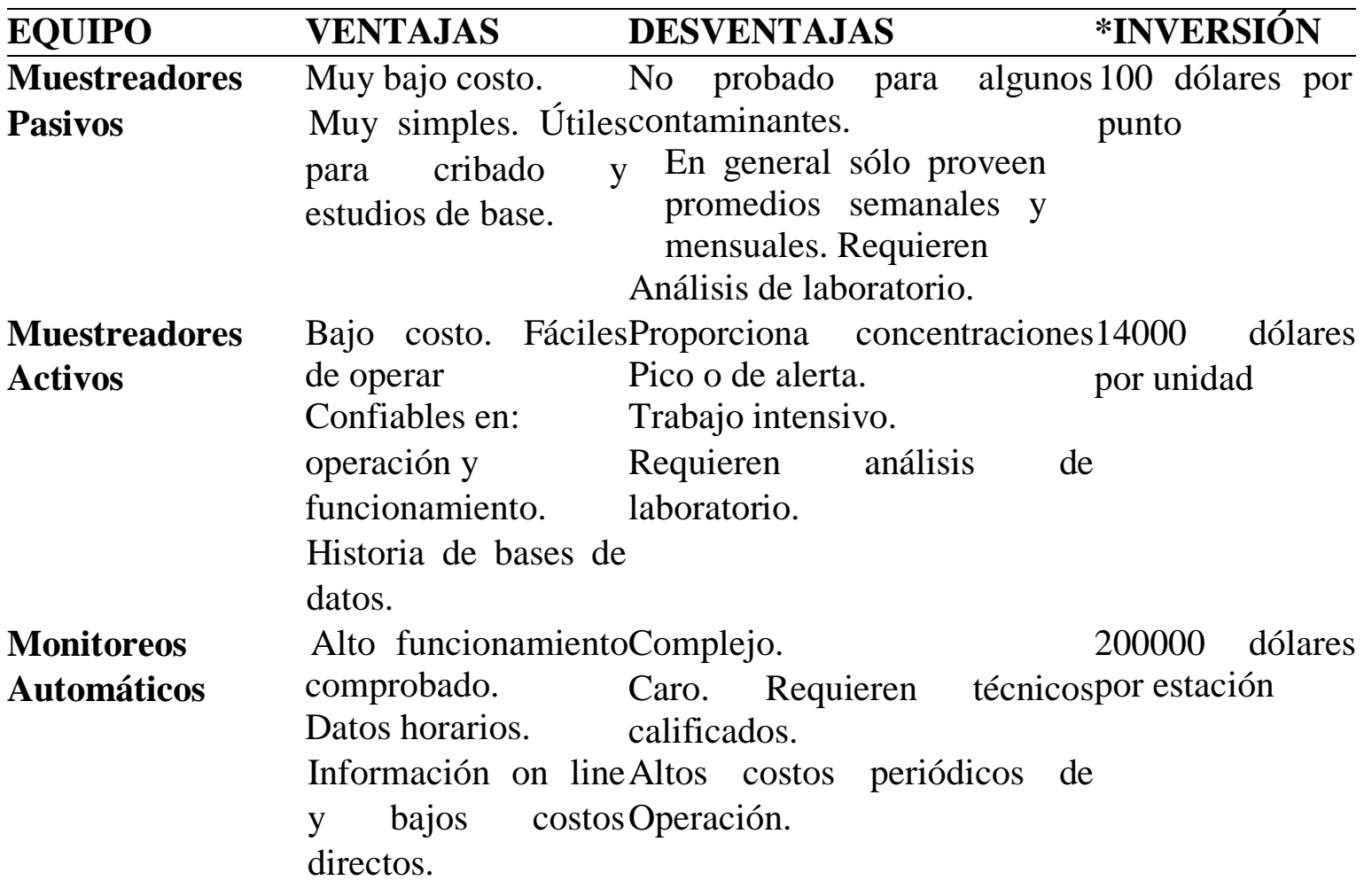

Nota: Resume las técnicas de monitoreo atmosférico recomendados

*Inversión estimada, indicado por Responsable del Monitoreo Atmosférico de Quito

Fuente: Texto de Gestión de calidad.2008 
Luego de mostrar las ventajas y desventajas de los equipos de monitoreo se presenta a continuación los métodos de análisis establecidos por la autoridad ambiental de control, la cual brinda información que permitirá al GAD Municipal implementar las técnicas de monitoreo en base a los requerimientos de medición.

Tabla 6. Métodos de medición de concentraciones de contaminantes.

\section{CONTAMINANTE NOMBRE, REFERENCIA Y DESCRIPCION DEL METODO}

Partículas Sedimentables Método Gravimétrico, mediante captación de partículas en envases abiertos.

Material Particulado (PM10) Método Gravimétrico, mediante muestreador de alto caudal o de bajo caudal.

Métodos Alternos: podrán ser también utilizados los denominados métodos de medición continua, tanto del tipo Microbalanza Oscilante como el tipo Atenuación Beta.

Material Particulado (PM2,5)Método Gravimétrico, mediante muestreador de bajo caudal.

Métodos Alternos: podrán ser también utilizados los denominados métodos de medición continua, tanto del tipo Microbalanza Oscilante como el tipo Atenuación Beta.

Dióxido de Azufre (SO2) Método de la Pararosanilina, Fluorescencia y Alterno: Podrá ser utilizado el método pasivo referido en la Norma Europea EN 13528-1:2002, EN 13528-2:2002, EN 13528-3:2002

Monóxido de Carbono (CO) Analizador infrarrojo no dispersivo (NDIR).

Ozono (O3) Quimiluminiscencia Fotómetro ultravioleta

Dióxido de Nitrógeno (NO2) Quimiluminiscencia

Método Alterno: Podrá ser utilizado el método pasivo referido en la Norma Europea EN 13528-1:2002, EN 13528-2:2002, EN 13528-3:2002

Benceno Adsorción TENAX ${ }^{\circledR} \quad \mathrm{Y}$ Cromatografía de gases/espectrometría de masas (GC/MS)

Nota: Se muestra los métodos de medición de los contaminantes criterios y no convencionales. 
Fuente: Libro VI Anexo 4. Norma de Calidad del Aire Ambiente o Nivel de Inmisión Elaborado por: Investigador, 2017

En base al costo de implementación y mantenimiento de los métodos de medición el GAD del Municipio de Riobamba determinará el tipo de monitoreo y los tiempos de implementación.

\section{Conclusiones}

- Los niveles de concentraciones de $\mathrm{SO}_{2}, \mathrm{NO}_{2}$, tolueno, xileno y etilbenceno en todos los años de monitoreo no registra un índice mayor al establecido en la Norma Ecuatoriana de Calidad del Aire, no supera los estándares establecidos en las Guías de la Organización Mundial para la Salud, sin embargo. En el caso del Benceno, se registró los máximos valores en el año 2009, con una concentración de $6,86 \mu \mathrm{g} / \mathrm{m}^{3}$ en la estación R-Norte-AME (noroeste de la ciudad) y de 8,36 $\mu \mathrm{g} / \mathrm{m}^{3}$, en la estación R-Sur-EMAPAR (sureste de la ciudad) la más alta del período de monitoreo, superando la norma que establece la Legislación Ecuatoriana que es de $5 \mu \mathrm{g} / \mathrm{m}^{3}$.

- Es necesario implementar nuevas estaciones de monitoreo según recomendaciones de organismos técnicos como la OPS, actividades y características de la zona.

- Según la población, niveles de contaminación y orografía de la ciudad de acuerdo a la OPS es conveniente implementar una estación automática.

\section{Agradecimiento}

Al Gobierno Autónomo Descentralizado del Cantón Riobamba, la Escuela Superior Politécnica de Chimborazo y al proyecto "Programa integrado de monitoreo para el control de la calidad del aire en la ciudad de Riobamba”, de la Universidad Nacional de Chimborazo, por contribuir con los datos y el modelo usado respectivamente.

\section{Referencias bibliográficas.}

[1]. Martinez, E. Díaz, Y. Contaminación atmosférica. Cuenca- España. Ediciones de la Universidad de Castilla. pp 13-87, 2004.

[2]. Haro A. et al, Characterization of the atmospheric dynamics in Riobamba city using de chaos theory, Atmospheric and Climate Sciences, Vol.5 No.4, pp. 441-449, October 2015.

[3]. Cocha L. Susana, Eemisión de gases y su relación en la calidad del aire de la zona urbana de la ciudad de Riobamba, Tesis, Universidad Técnica de Ambato, 2017.

[4].Secretaría del Ambiento, Informe de la calidad del aire, 2017.

[5].Palacios Espinoza Elvira, Molina Claudia Espinoza, (2014) Contaminación del aire exterior. Cuenca - ecuador, 2009- 2013. Posibles efectos en la salud, Revista de la Facultad de Ciencias Médicas, Volumen 32, Cuenca, Ecuador. 
file://C:/Users/Arquimides\%20Haro/Downloads/883-2784-1-PB.pdf, 27 de enero de 2017.

[6]. Alarcon, E. Callarotti, et al. (2000). Modelos Matemáticos en Ingeniería Moderna. Caracas- Venezuela. Universidad Central de Venezuela. pp301-303.

[7]. Ministerio del Ambiente, Plan nacional de la calidad del aire, [2010].

[8].Figuruelo, J.E. Davila ,M.M. (2004). Química Física del Ambiente y de Procesos Medio Ambientales. Barcelona - España. Reverte. pp. 1-104.

[9]. Trozzi C., Piscitello E., et. al. (2003). Modeling area, line and point sources for ISC model. methodology, computer interface and case studies, Emission inventories, Roma -Italy. pp. 1-27. http://nlquery.epa.gov/epasearch. 2015-09-25

[10]. BOLETÍN DE LA ACADEMIA NACIONAL DE MEDICINA DE MÉXICO, La contaminación del aire y los problemas respiratorios. Rev. Fac. Med. (Méx.) [online]., vol.58, n.5, pp.44-47. ISSN 2448-4865, 2015.

[11]. Rosa María Gómez, Ing, Msc1, Paola Andrea Filigrana, Mag. Epidemiol1, Fabián Méndez,Descripción de la calidad del aire en el área de influencia del Botadero de Navarro, Cali, Colombia, Colombia Médica, 2008.

[12]. Aeromod, EPA, (2004) Description of model formulation. EPA-454/R-03-004, Office of Air Quality Planning and Standards, p. 91.

[13]. Geografía de Ecuador, https://es.wikipedia.org/ wiki/Geograf\%C3\%A Da_de_ Ecuador. Revisado en línea, 3 de junio de 2015.

[14]. Boublel, R. Fox Donal, et al, Fundamentals at Air Pollution. 3a ed. NewYork Estados Unidos. Academic Press. pp. 243-362, . 1990. .

[15]. Amanda H., et al, (2006), Applied Atmospheric Dynamics, John Wyle and Sons, England.

[16]. Cian J Desmond, Simon Watson, (2014) A study of stability effects in forested terrain, Journal of Physics: Conference Series 555, 2014.

[17]. Panofsky Ha And Dutton Ja, (1984) Atmospheric Turbulence - Models and Methods for Engineering Applications. New York: Wiley, 1984.

[18]. Wyngaard, J. C., (1998) Convection viewed from a turbulence perspective. Buoyant Convection in Geophysicsl Flows, Advanced Science Institute Series, 23- 39. 


\section{PARA CITAR EL ARTÍCULO INDEXADO.}

Perugachi Cahueñas, N., \& Cocha Telenchana, L. (2019). Rediseño de la red de monitoreo atmosférico para control y vigilancia de la calidad del aire en la ciudad de Riobamba. Ciencia Digital, 3(3.2), $172-192$. https://doi.org/10.33262/cienciadigital.v3i3.2.723

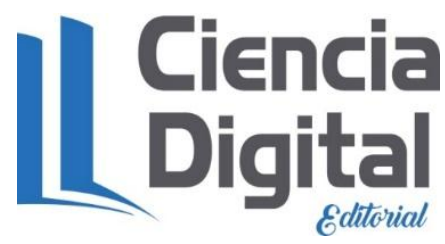

El artículo que se publica es de exclusiva responsabilidad de los autores y no necesariamente reflejan el pensamiento de la Revista Ciencia Digital.

El artículo queda en propiedad de la revista y, por tanto, su publicación parcial y/o total en otro medio tiene que ser autorizado por el director de la Revista Ciencia Digital.
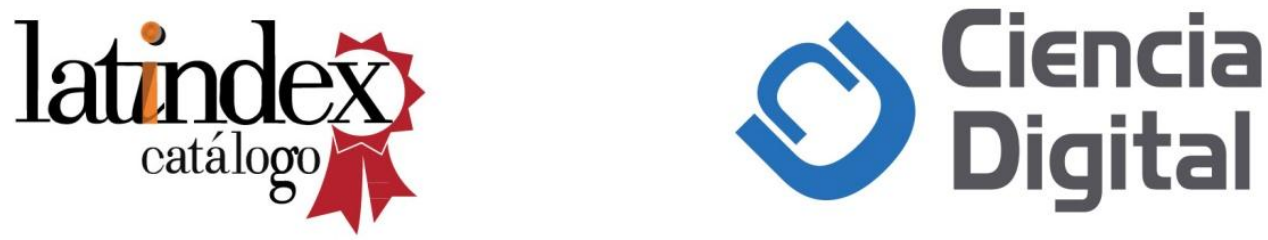